\title{
Detection and sequence analysis of the DNA repair gene RAD51 in the Korean spider Callobius koreanus (Amaurobiidae)
}

J.Y. Kim and Y.C. Park

Division of Forest Science, College of Forest \& Environmental Sciences,

Kangwon National University, Chuncheon, Republic of Korea

Corresponding author: Y.C. Park

E-mail: parky@kangwon.ac.kr

Genet. Mol. Res. 14 (4): 14646-14648 (2015)

Received July 22, 2015

Accepted September 30, 2015

Published November 18, 2015

DOI http://dx.doi.org/10.4238/2015.November.18.28

ABSTRACT. We identified a partial sequence (483 bp) of the RAD51 gene from the Korean spider Callobius koreanus. Sequence variation was found at one position during alignment with the human RAD51 gene sequence. This partial sequence included the region corresponding to exon 4 in the human RAD51 gene, which encodes 39 amino acids. These results show that the RAD51 gene is highly conserved between human and spiders.

Key words: Callobius koreanus; RAD51; Exon, DNA repair gene; Amaurobiidae 


\section{INTRODUCTION}

The RAD51 gene encodes the RAD51 protein, which is essential for repairing damaged DNA. In humans, the RAD51 gene is located between base pairs 40,694,774 and 40,732,158 on the long (q) arm of chromosome 15, which corresponds to a length of 37,385 bp (NCBI's genome browser Map Viewer-Homo sapiens Annotation Release 107). The protein encoded by this gene is a member of the RAD51 protein family and is highly similar to bacterial $\operatorname{Rec} A$ and Saccharomyces cerevisiae RAD51, which are involved in the homologous recombination and repair of DNA (Shinohara et al., 1992; Morais Jr et al., 1998). Characteristics of RAD51 are relatively well-known in mammals, amphibians, and birds (Maeshima et al., 1995; Takata et al., 2002; Venkitaraman, 2002), but are poorly reported in invertebrates, especially spiders. In this study, we identified and analyzed a partial sequence of the RAD51 gene from a Korean spider species, Callobius koreanus.

\section{MATERIAL AND METHODS}

Detailed methods used to obtain a partial sequence of the RAD51 gene from $C$. koreanus are provided in Table S1. The obtained RAD51 gene sequence was compared with other homologous sequences deposited in GenBank using BLASTN2.2.31+ (Zhang et al., 2000). The amino acid sequence translated from the RAD51 gene was compared with other homologous protein sequences in the PDB protein database (NCBI) using BLASTP 2.2.32+ (Altschul et al., 1997). To compare homology, sequences were aligned using the Clustal-W program found in Geneious Pro 5.5.9 (Biomatters, Auckland, New Zealand).

\section{RESULTS AND DISCUSSION}

We obtained a partial RAD51 gene sequence of 483 bp (KT325925) from the Korean spider $C$. koreanus. Using a BLAST search in GenBank, the sequences with most homology to $C$. koreanus RAD51 were found to be those belonging to the $H$. sapiens RAD51 genes (NG_012120 and AY196785) with a query cover of $100 \%$ and identity of $99 \%$. The aligned region spans positions 15,920 to 16,402 on the Homo sapiens RAD51 gene (NG_012120) (Figure 1). The aligned sequences of the two genes differed at only one position ( $T<->G$ at position 15,984). The $H$. sapiens RAD51 gene contains 10 exons, which encode the 339 amino acid sequence of RAD51 recombinase (Park et al., 2008). Of these, exon 4 is located from position 16,017 to 16,134 within the aligned sequences and encodes 39 amino acids (Figure 1). In a BLAST search of the PDB protein database (NCBI), the sequence derived from 39 amino acids translated from $117 \mathrm{bp}$ of the $C$. koreanus RAD51 gene in the exon 4 region was found to be homologous to RAD 51 sequences reported in various vertebrates including primates, birds, and rodents. Although the results obtained in this study depend on the partial sequence of $C$. koreanus RAD51, they suggest that this sequence might be well conserved between human and spiders. 


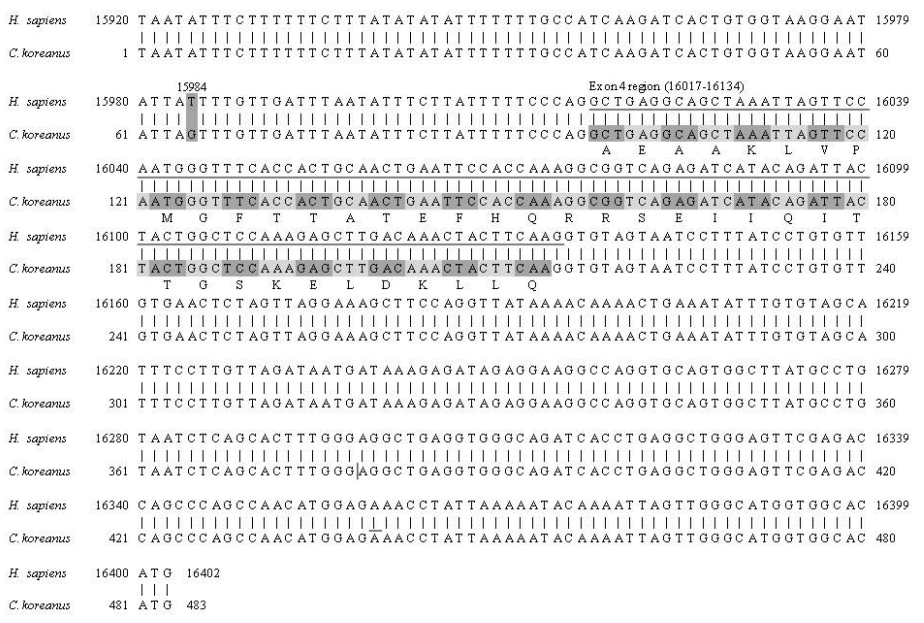

Figure 1. Sequence analysis of the Callobius koreanus RAD15 gene. The gene sequence was aligned from positions 15,920 to 16,402 on the Homo sapiens RAD51 gene sequence (NG_012120). All positions of the sequences are well conserved except for position 15,984. The exon 4 region of $H$. sapiens, which is located between positions 16,017 and 16,134 , is underlined. One-letter amino acid symbols according to the IUPAC-IUB single-letter amino acid codes are denoted for each codon in the corresponding region of the C. koreanus RAD51 gene.

\section{Conflicts of interest}

The authors declare no conflict of interest.

\section{ACKNOWLEDGMENTS}

Research supported by the Basic Science Research Program through the National Research Foundation of Korea (NRF) funded by the Ministry of Education (\#NRF-2012R1A1A4A01015001).

\section{Supplementary material}

\section{REFERENCES}

Altschul SF, Madden TL, Schäffer AA, Zhang J, et al. (1997). Gapped BLAST and PSI-BLAST: a new generation of protein database search programs. Nucleic Acids Res. 25: 3389-3402.

Maeshima K, Morimatsu K, Shinohara A and Horii T (1995). RAD51 homologues in Xenopus laevis: two distinct genes are highly expressed in ovary and testis. Gene 160: 195-200.

Morais Jr MA, Vlcková V, Fridrichová I, Slaninová M, et al. (1998). Effect of bacterial recA expression on DNA repair in the rad51 and rad52 mutants of Saccharomyces cerevisiae. Genet. Mol. Biol. 21: 3-9.

Park JY, Yoo HW, Kim BR, Park R, et al. (2008). Identification of a novel human Rad51 variant that promotes DNA strand exchange. Nucleic Acids Res. 36: 3226-3234.

Shinohara A, Ogawa H and Ogawa T (1992). Rad51 protein involved in repair and recombination in S. cerevisiae is a RecA-like protein. Cell 69: 457-470.

Takata M, Tachiiri S, Fujimori A, Thompson LH, et al. (2002). Conserved domains in the chicken homologue of BRCA2. Oncogene 27: 1130-1134.

Venkitaraman AR (2002). Cancer susceptibility and the functions of BRCA1 and BRCA2. Cell 108: 171-182.

Zhang Z, Schwartz S, Wagner L and Miller W (2000). A greedy algorithm for aligning DNA sequences. J. Comput. Biol. 7: 203-214. 